\title{
Activin A contributes to the development of hyperoxia-induced lung injury in neonatal mice
}

\author{
Rebecca Lim ${ }^{1,2}$, Ruth Muljadi', Eugenia Koulaeva², Patricia Vosdoganes², Siow Teng Chan', Rutu Acharya',
} Seshini Gurusinghe ${ }^{1}$, Olli Ritvos ${ }^{3}$, Arja Pasternack ${ }^{3}$ and Euan M. Wallace ${ }^{1,2}$

\begin{abstract}
BACKGROUND: Bronchopulmonary dysplasia (BPD) is one of the leading causes of morbidity and mortality in babies born prematurely, yet there is no curative treatment. In recent years, a number of inhibitors against TGF $\beta$ signaling have been tested for their potential to prevent neonatal injury associated with hyperoxia, which is a contributing factor of BPD. In this study, we assessed the contribution of activin A-a member of the TGF $\beta$ superfamily - to the development of hyperoxia-induced lung injury in neonatal mice.
\end{abstract}

METHODS: We placed newborn C57Bl6 mouse pups in continuous hyperoxia $\left(85 \% \mathrm{O}_{2}\right)$ to mimic many aspects of BPD including alveolar simplification and pulmonary inflammation. The pups were administered activin A receptor type IIB-FC antagonist (ActRIIB-FC) at $5 \mathrm{mg} / \mathrm{kg}$ or follistatin at $0.1 \mathrm{mg} / \mathrm{kg}$ on postnatal days 4, 7, 10, and 13.

RESULTS: Treatment with ActRIIB-FC and follistatin protected against hyperoxia-induced growth retardation. ActRIIB-FC also reduced pulmonary leukocyte infiltration, normalized tissue: airspace ratio and increased septal crest density. These findings were associated with reduced phosphorylation of Smad3 and decreased matrix metalloproteinase (MMP)-9 activity.

CONCLUSION: This study suggests that activin A signaling may contribute to the pathology of bronchopulmonary dysplasia.

P remature birth refers to birth before $37 \mathrm{wk}$ of gestation. There are a large number of medical complications associated with being born prematurely, many of which are a consequence of life-saving interventions, including bronchopulmonary dysplasia (BPD). Historically, BPD was originally described in 1967, as a form of chronic lung disease observed in premature babies, resulting from the injurious effects of high oxygen tension and airway pressure used during mechanical ventilation (1). This form of lung disease is commonly referred to as "old BPD". Today, this is rare due to gentler ventilation strategies and the use of surfactant and antenatal steroids. Instead, a "new BPD" has emerged, defined as the requirement of supplemental oxygen at $36 \mathrm{wk}$ postconceptional age (2). The pathological hallmarks are suggestive of arrested septation and alveolarisation triggered by inflammation, corticosteroids, and hyperoxia (3).
There is currently no cure for BPD and asides from ventilatory support, clinical management involves the use of steroids, which is associated with an increased incidence of cerebral palsy. There is thus a need for new therapeutic approaches.

In the past decade, much light has been shed on the contribution of TGF $\beta$ signaling to neonatal lung development and injury. TGF $\beta 1$ has been shown to be important for the survival and repair of type II alveolar epithelial cells (AEC2) (4). Specifically, TGF $\beta 1$ treatment of hyperoxia damaged AEC2 improved cell survival, migration, and secretion of proangiogenic ligands such as vascular endothelial growth factor (VEGF) and matrix proteins such as fibronectin. However, inhibition of TGF $\beta$ signaling by curcumin and PPAR $\gamma$ agonist, rosiglitazone, were protective against hyperoxia-induced lung injury in neonatal rat models of BPD $(5,6)$. These reports point to the need for better understanding of TGF $\beta$ signaling within the context of BPD.

Activin A, a member of the TGF $\beta$ superfamily, has been reported to contribute to adult lung disease. In adult animals, overexpression of activin $\mathrm{A}$ in the lungs results in an alteration in the composition of the lung epithelial cell populations where ciliated cells dominate, with fewer AEC2 (7). These changes result in a compromise to lung compliance and a significant reduction in surfactant protein C-expressing AEC2. In another study, blocking activin A using follistatin following in bleomycin challenge, prevented lung inflammation and fibrosis (8). While these studies support a role for activin A in adult inflammatory and fibrotic lung injury, its role in neonatal lung injury, repair and development has never been previously studied.

In this study, we exposed newborn mice to hyperoxia (85\% $\mathrm{O}_{2}$ ) and administered either soluble ActRIIB-Fc ( $5 \mathrm{mg} / \mathrm{kg}$ ) or follistatin $(0.1 \mathrm{mg} / \mathrm{kg})$ twice weekly to determine the contribution of activin A signaling to the pulmonary phenotype. We assessed changes to lung remodeling, activin A levels, Smad signaling and markers of lung injury including leukocyte infiltration, cell death, and proliferation.

\section{RESULTS}

ActRIIB-Fc and Follistatin Treatment Improved Bodyweights Hyperoxia reduced bodyweights of pups as previously reported. The bodyweights of animals from the hyperoxia

${ }^{1}$ The Ritchie Centre, MIMR-PHI Institute of Medical Research, Victoria, Australia; ${ }^{2}$ Department of Obstetrics and Gynecology, Monash University, Victoria, Australia; ${ }^{3}$ Department of Bacteriology and Immunology, Haartman Institute, University of Helsinki, Helsinki, Finland. Correspondence: Rebecca Lim (Rebecca.Lim@monash.edu) 
a

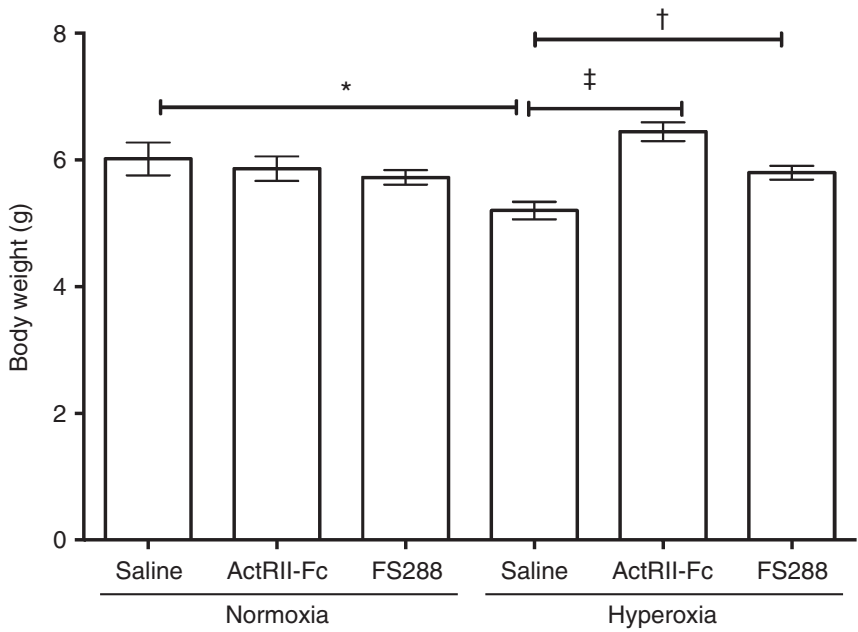

b

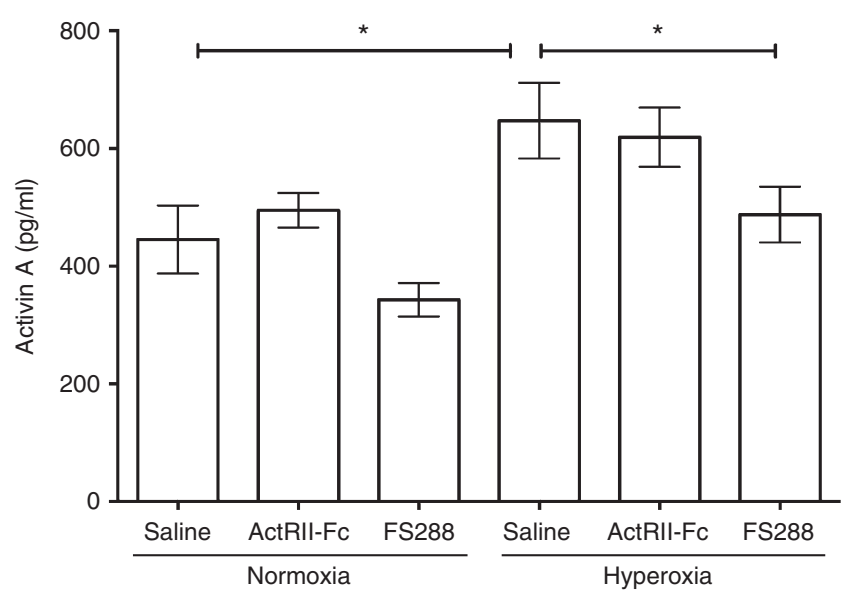

Figure 1. Hyperoxia reduced bodyweights of neonatal mouse pups. Hyperoxia reduced bodyweights of developing pups. They are significantly lighter at postnatal day day 14 compared to normoxia pups $\left(\mathbf{a},{ }^{*} P<0.05\right)$. Administration of ActRIIB-Fc and FS288 significantly increased bodyweights of hyperoxia-exposed pups $\left({ }^{\dagger} P<0.001,{ }^{\ddagger} P<0.0001\right)$. Activin A levels were elevated in hyperoxia animals, which was reduced following FS288 treatment $(\mathbf{b}, * P<0.05)$.

+ saline cohort were significantly lower compared to the normoxia + saline cohort at postnatal day 14 (Figure 1a). Treatment with ActRIIB-Fc and follistatin significantly improved the bodyweights of pups subjected to hyperoxia such that they were not significantly different to the normoxia treatment groups. Hyperoxia was associated with increased activin levels (Figure 1b, $P<0.05$ ), which remained unchanged by ActRIIB-Fc treatment but significantly reduced by follistatin $(P<0.05)$.

\section{ActRIIB-Fc Treatment Improves Septation}

Hyperoxia increased Smad3 phosphorylation as previously reported by Dasgupta et al. (9) (Figure 2a, $P<0.05$ ). This was significantly reduced by ActRIIB-Fc and follistation $(P<0.01)$. Representative western blots are provided in Figure 2b. Typical of hyperoxia exposure, we saw a reduction in tissue: airspace ratio (Figure 3a, $P<0.01$ ) as increased oxygen tension induced alveolar simplification. Treatment with ActRIIB-Fc significantly increased tissue: airspace ratio in hyperoxia-treated animals $(P<0.0001)$, suggesting that there was some prevention of alveolar simplification.

Smad 2/3 signaling is increased in mouse models of hyperoxia-induced lung injury (5) as well as sheep models of antenatal inflammation-associated lung injury (10). Given the effects of ActRIIB-Fc treatment on improving tissue: airspace ratio and reducing phosphorylation of Smad 3, we sought to assess the effects of ActRIIB-Fc and follistatin on septation. Secondary septal crest density was thus determined by morphometric analysis. We confirmed that hyperoxia significantly reduced septal crest density (Figure 3a, $P<0.001)$. Administration of ActRIIB-Fc increased septation in the hyperoxia-treated animals $(P<0.0001)$ while follistatin treatment had no significant impact on septation. Representative images of septal crest imaging are shown in Figure 3c.

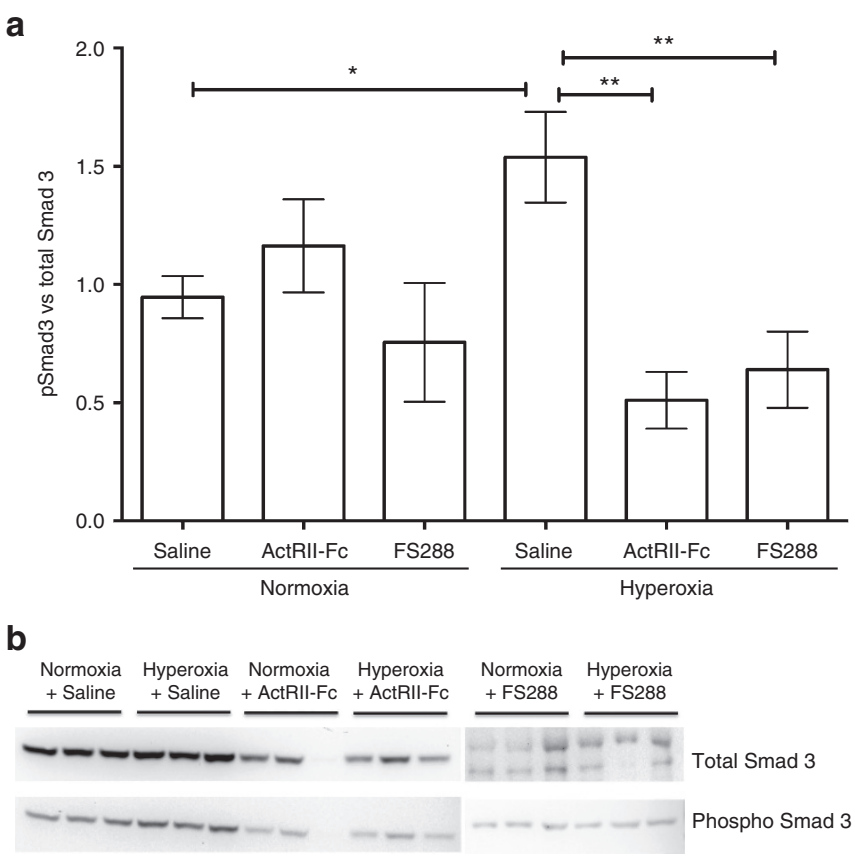

Figure 2. Treatment with ActRIIB-Fc and follistatin reduced hyperoxia-induced Smad 3 phosphorylation. Hyperoxia significantly increased Smad 3 phosphorylation $\left(\mathbf{a},{ }^{*} P<0.05\right)$. This was reduced in ActRIIB-Fc- and FS288-treated animals ( $\left.{ }^{* *} P<0.01\right)$. Representative western blots are shown (b).

\section{ActRIIB-Fc Treatment Reduced Pulmonary Leukocyte Infiltration} and IL-6 Production

Unsurprisingly, hyperoxia increased the recruitment of leukocytes in the lungs as determined by CD45 immunohistochemistry (Figure 4a, $P<0.05$ ). This was significantly reduced by ActRIIB-Fc treatment $(P<0.01)$ where the percentage of $\mathrm{CD} 45^{+}$cells was reduced to numbers comparable to that of normoxia + saline animals. Given that ActRIIB-Fc treatment 


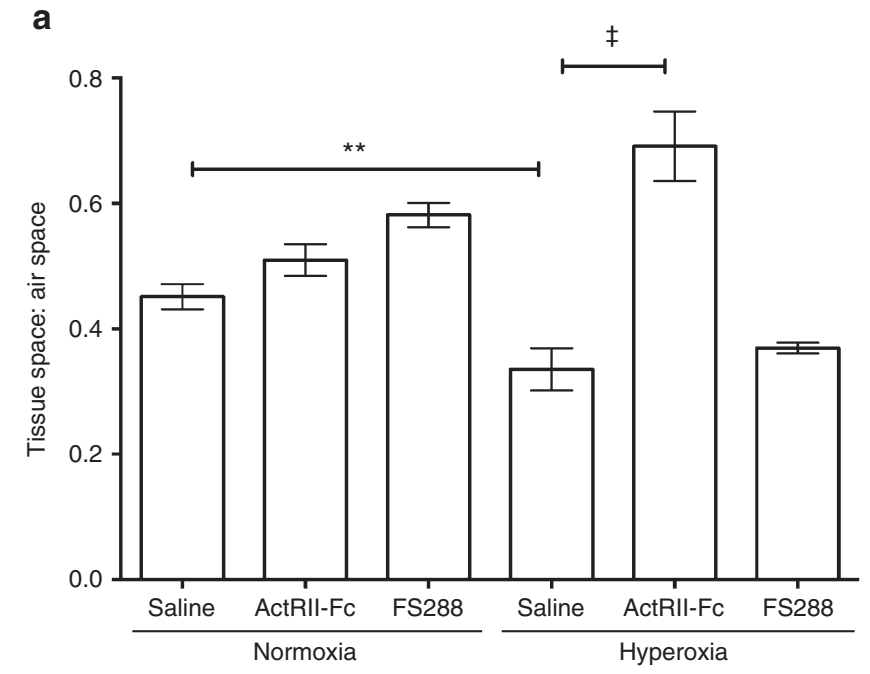

b

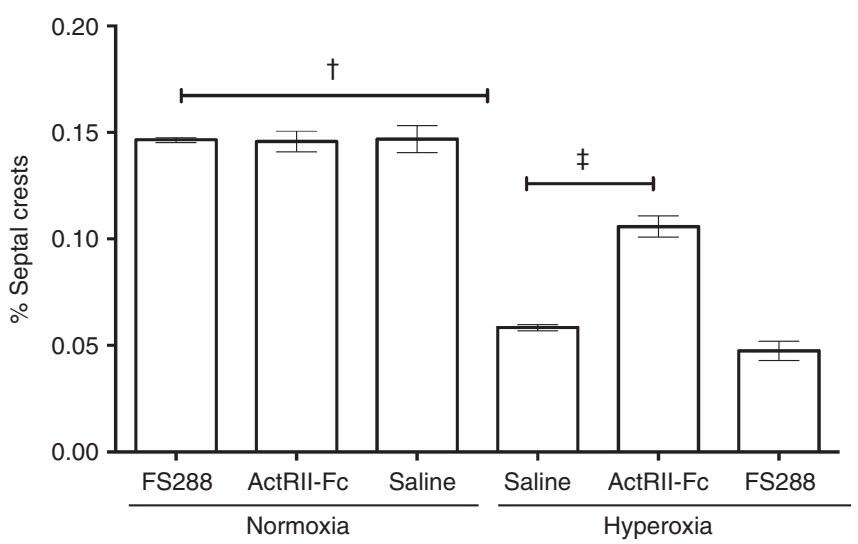

C

Saline

ActRII-FC
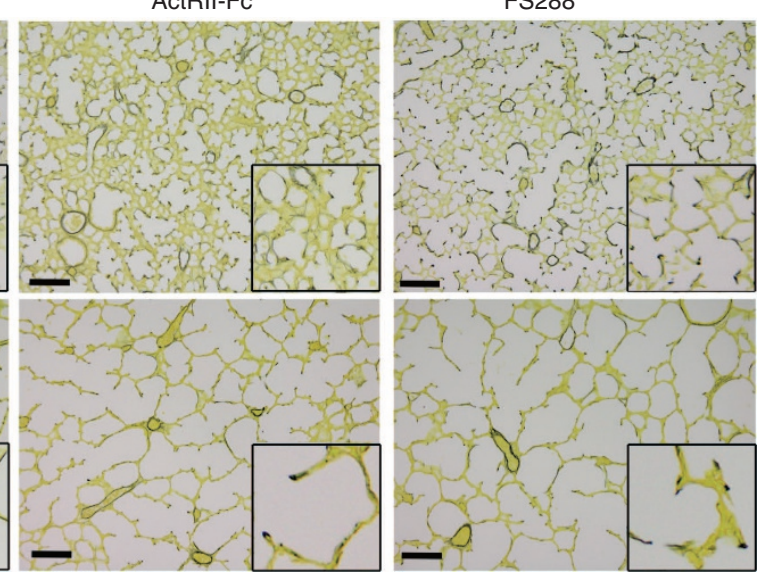

Figure 3. Changes in tissue: airspace ratio and secondary septation. Tissue: airspace ratio was reduced by hyperoxia (a, $\left.{ }^{*} P<0.01\right)$. Treatment with

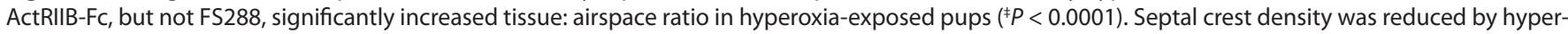
oxia $\left(\mathbf{b},{ }^{\dagger} P<0.001\right)$ but prevented by ActRIIB-Fc treatment $\left({ }^{\ddagger} P<0.0001\right)$. Representative images of Hart's-stained sections used for septal crest scoring are shown (c). Images were taken at $200 \times$ magnification. Scale bars: $100 \mu \mathrm{m}$. Inset panels show Hart's stain accumulated at secondary septal crests.

had the greatest impact on immune cell numbers, we further assessed the effect of this treatment on macrophage and neutrophil numbers. Here we observed no significant effect of ActRIIB-Fc treatment on numbers of lung macrophages (Figure 4b) or neutrophils (Figure 4c). However, pulmonary levels of IL-6 were increased in the neonatal mouse pups exposed to hyperoxia as previously reported (11) (Figure 4d, $P<0.01)$. This was prevented by ActRIIB-Fc treatment $(P<0.0001)$.

\section{ActRIIB-Fc Treatment Prevents Pathological Lung Remodeling}

We next asked if improvements in tissue: airspace ratio was due to modulation of matrix metalloproteinase activity since matrix metalloproteinase (MMP)-2 and -9 , were previously reported to contribute to lung remodeling during hyperoxia (12). Here we found at postnatal day 14, that hyperoxia had no significant effect on MMP-2 activity in the lungs (Figure 5a), but significantly increased MMP-9 activity (Figure $5 b, P<0.001$ ). This was significantly reduced by ActRIIB-Fc treatment $(P<0.01)$.

\section{ActRIIB-Fc Treatment Does Not Affect AEC2 Proliferation}

Further, we asked if ActRIIB-Fc treatment promoted proliferation and of the actively proliferating cells, how many were AEC2. To assess this, we performed double immunofluorescence for SPC and Ki67. Representative images of immunofluorescence are provided in Figure 6a. We found that hyperoxia increased the percentage of AEC2 cells, as was recently reported (13) (Figure $6 \mathbf{b}, P<0.01$ ). This was not changed by ActRIIB-Fc by postnatal day 14. Assessing total Ki67+ cells, we also observed that hyperoxia increased cell proliferation (Figure 6c, $P<0.0001$ ) - an effect that was inhibited by ActRIIB-Fc treatment $(P<0.0001)$. Colocalization of these markers indicates that ActRIIB-Fc treatment did not negatively impact AEC2 proliferation (Figure 6d).

\section{DISCUSSION}

With ever-growing improvements in obstetric and perinatal medicine, viable births are being delivered at increasing prematurity. One of the major complications of premature births 


\section{Articles | Limetal.}
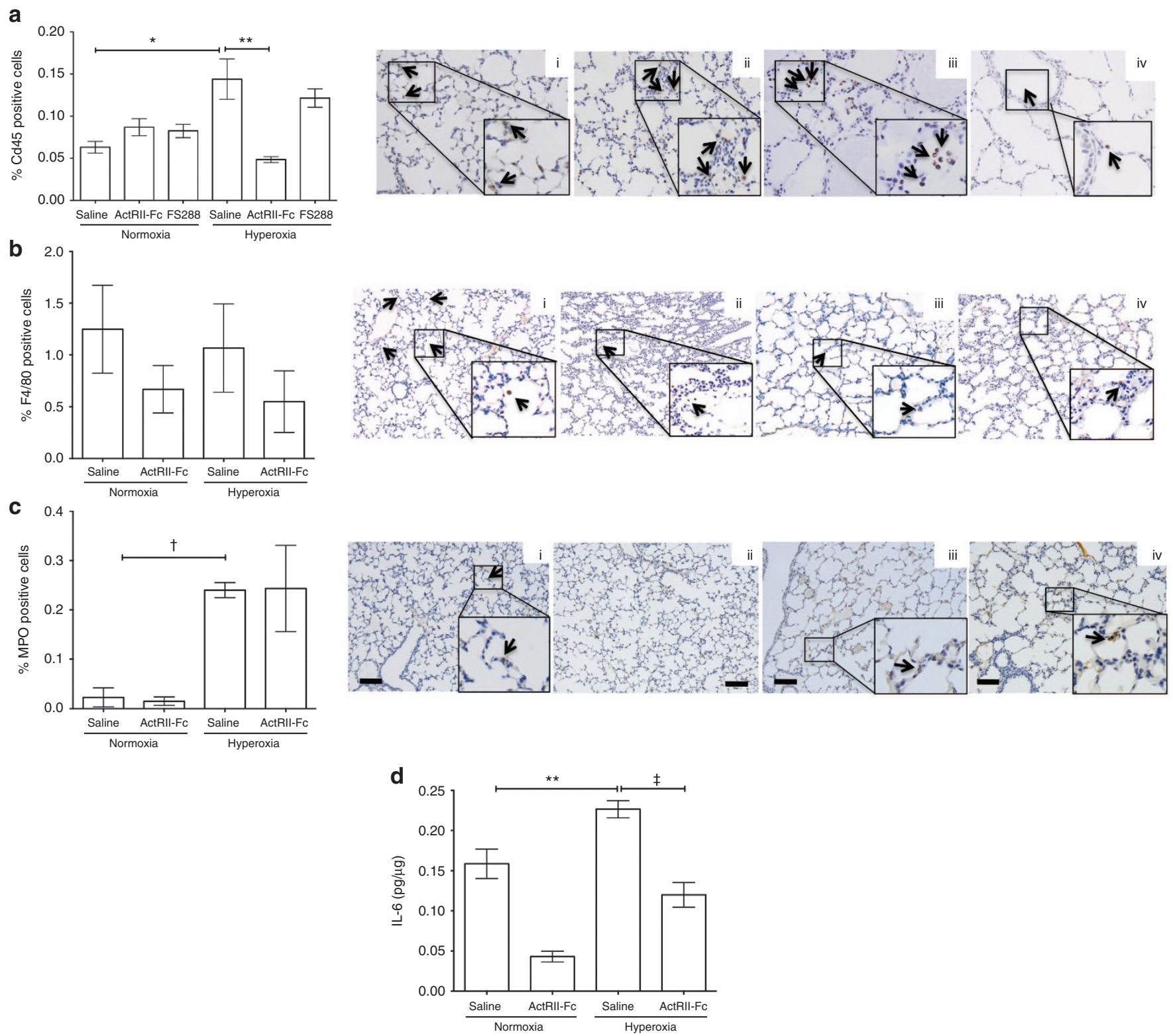

Figure 4. Antagonism of activin A signaling offered limited protection against hyperoxia-induced inflammation. Hyperoxia increased leukocyte numbers and this was reduced by ActRIIB-Fc treatment $\left(\mathbf{a},{ }^{*} P<0.05,{ }^{* *} P<0.01\right)$. Representative images were taken at $400 \times$ magnification. Hyperoxia did not change macrophage numbers (b, representative images taken at $200 \times$ magnification) but increased neutrophil numbers (c, representative images were taken at 200x magnification). Scale bars $100 \mu \mathrm{m}$. Black arrows point at positive stained cells. (i) normoxia + saline; (ii) normoxia + ActRBIIB-Fc; (iii) hyperoxia + saline; (iv) hyperoxia + ActRIIB-Fc IL-6 levels in lung homogenates were elevated in hyperoxia-treated animals $\left(\mathbf{d},{ }^{* *} P<0.01\right)$ and reduced with ActRIIB-Fc treatment ( $\left.{ }^{P} P<0.0001\right)$.

is BPD, a major cause of morbidity and mortality in premature babies. There is still no cure for BPD and it remains a clinical problem. Here we evaluate the contribution of activin A in the development of hyperoxia-induced lung injury using a neonatal mouse model of BPD. We found that both ActRIIB-Fc and FS288 were effective in protecting against hyperoxia-associated weight loss. Activin A levels and Smad 3 phosphorylation were elevated in the lungs of neonatal mice exposed to hyperoxia. Activin A levels were normalized by follistatin but phosphorylation of Smad 3 was most diminished by ActRIIB-Fc. ActRIIB-Fc was more effective in reducing leukocyte infiltration. These findings were accompanied by increased septal crest density and reduction in MMP-9 activity, suggesting that activin A or related members of the TGF $\beta$ superfamily may place a role in the deleterious lung remodeling during hyperoxia.

TGF $\beta$ signaling plays an important role during lung development where TGF $\beta$ levels are developmentally regulated. Exogenous TGF $\beta$ can promote survival and restoration of hyperoxia-depleted autocrine VEGF secretion in AEC2 and TGF $\beta$-mediated Smad signaling is important for the maintenance of this cell population (4). Conditional knockout of TGF $\beta$ II receptor (T $\beta$ RII) in the lung epithelium resulted in retardation of postnatal alveolarisation with marked reduction in type I alveolar epithelial cells (AEC1) (14). Similar findings 
a

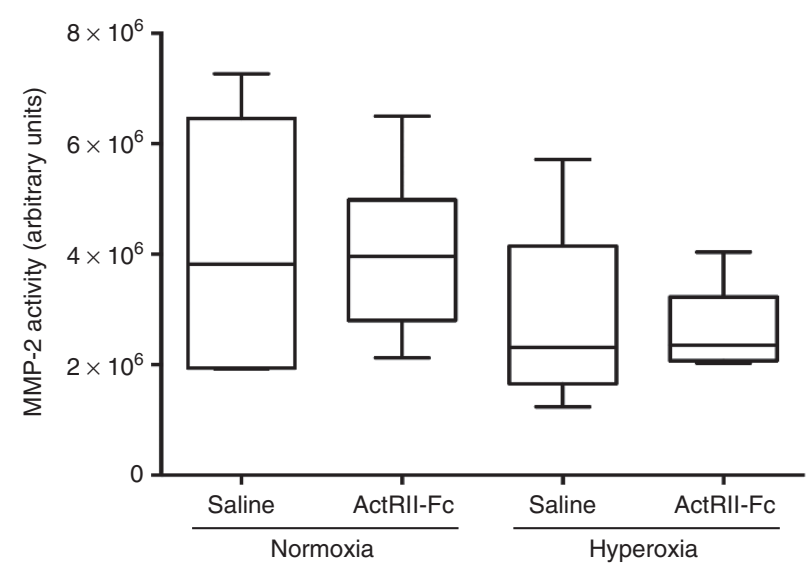

b

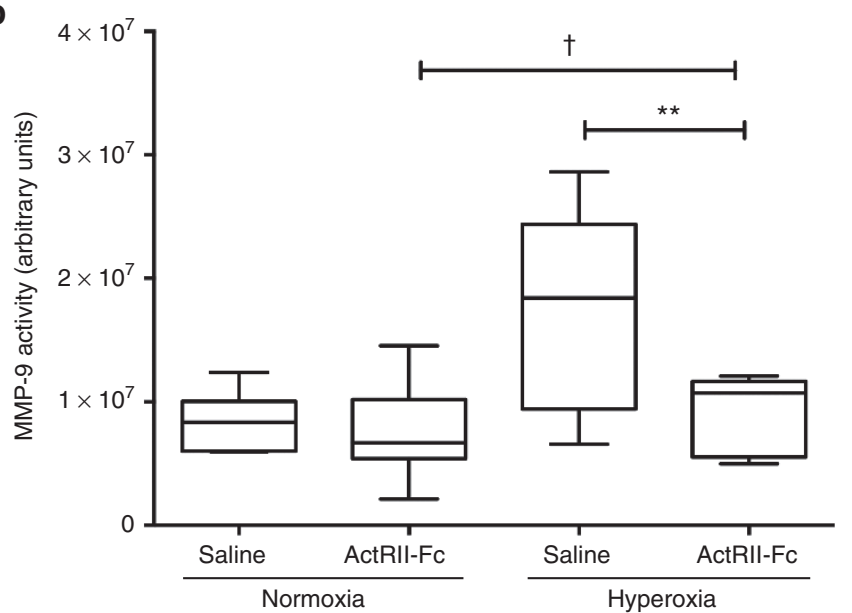

Figure 5. Changes to metalloproteinase-2 and -9 activity. Activity of matrix metalloproteinase (MMP)-2 did not differ between experimental groups (a). Hyperoxia increased MMP-9 activity in lung lysates $\left(b,{ }^{\dagger} P<0.001\right)$. This was increased by ActRIIB-Fc $(* * P<0.01)$.

a

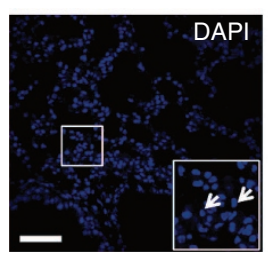

b

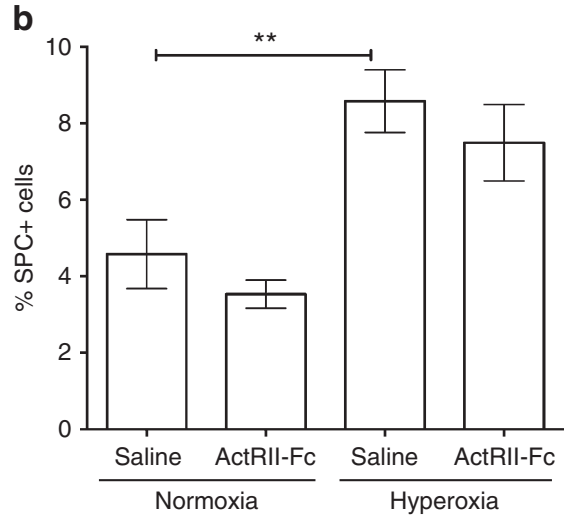

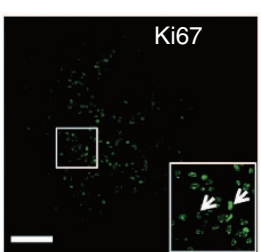

c
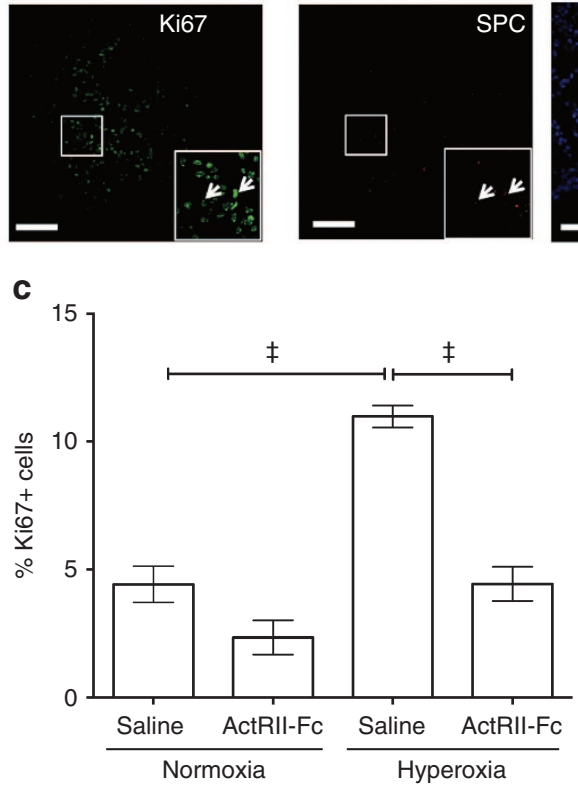

$k$

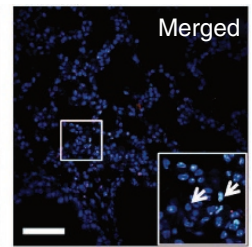

d

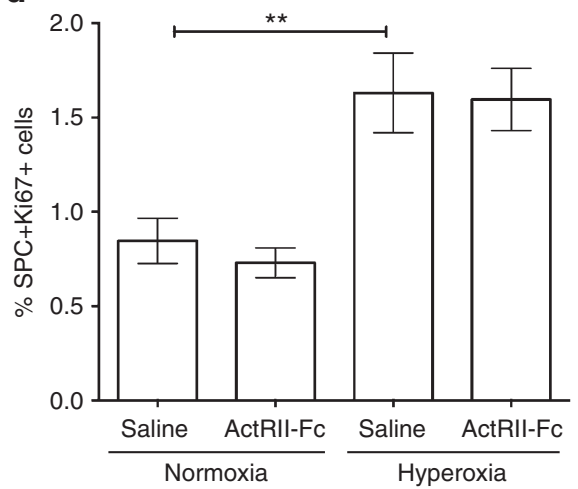

Figure 6. Hyperoxia increases AEC2 proliferation and is not affected by ActRIIB-Fc treatment. Representative images were taken at $400 \times$ magnification and white arrows point to double-positive cells (a). Scale bars $50 \mu \mathrm{m}$. Hyperoxia significantly increased SPC+ cells $\left(\mathbf{b},{ }^{* *} P<0.01\right)$ and total cell proliferation $\left(\mathbf{c}_{,}{ }^{\ddagger} P<0.0001\right)$. This effect was reduced in ActRIIB-Fc treated mice $\left({ }^{\ddagger} P<0.0001\right)$. Hyperoxia increased proliferation of type II alveolar epithelial cells $\left(\right.$ d, $\left.{ }^{* * P}<0.01\right)$, which was unaltered by ActRIIB-Fc.

were reported in Smad3-deficient mice, where pronounced lung alveolarisation was observed at postnatal days 7-28 (15). These reports thus indicate that $\mathrm{Smad} 3$ is an important positive regulatory element in normal lung development. During hyperoxic neonatal lung injury, however, inhibition of TGF $\beta$ signaling can be beneficial. In a neonatal hyperoxia mouse model, alveolar arrest was shown to correspond strongly to dysregulation expression and immunohistochemical localization of ALK- $1,-3$, and -6 and TGF $\beta$ receptor, as well as Smads 1,3 and 4, in lung tissues. The same study showed that TGF $\beta$ signaling increased in lung epithelial cells upon exposure to hyperoxia in vivo and in vitro (16).
Given the nature of activin A, having been described as having both pro- and anti-inflammatory effects, and sharing common Smad signaling intermediates with TGF $\beta 1$, an assessment into the role of activin A in hyperoxic lung injury is warranted. This is the first study to evaluate the contribution of activin A signaling to the development of hyperoxia-induced lung injury and the first study to use ActRIIB-Fc as an experimental treatment for lung injury.

Follistatin is a naturally occurring protein that binds strongly to activins (Kd 50-900mP) and inhibits their activin bioactivity. Follistatin can also bind to other members of the TGF $\beta$ superfamily including BMP- $4,-6$, and -7 , albeit at 
lower affinities (17). Notably, ActRIIB-Fc binds most strongly to activin $\mathrm{A}$, but it is also able to bind to other members of the TGF $\beta$ superfamily such as GDF- 8 and -11 , as well as BMP-2 and -7, with up to 100-fold lower affinity (18). Activin A was previously reported to induce proinflammatory cytokines such as IL-6, TNF $\alpha$, IL-1 $\beta$, and iNOS in macrophages in vitro (19). In other studies, activin A suppressed IL-6 signaling (20), macrophage-derived nitric oxide (21), dendritic cell maturation (22), induction of regulatory T cells $(23,24)$. Activin A has also been implicated in matrix remodeling and promotion of a Th2 immune response for wound healing $(25,26)$. It is perhaps unsurprising then, that the antagonism of activin A signaling using ActRIIB-Fc or FS288 did not wholly ameliorate all pathological aspects of hyperoxiainduced lung injury. It was, however, unexpected that follistatin treatment, but not ActRIIB-Fc, reduced activin A levels since one would expect that both treatment regimes target the downstream effects of activin A. This limitation could be addressed in subsequent studies by measuring free activin and/or activin activity levels instead of total activin A, as performed in our current study.

The reduction in pulmonary leukocyte infiltration and levels of proinflammaory cytokine IL-6 does suggest, however, that inhibition of activin A signaling during hyperoxia may be protective either by reducing local inflammation brought on by hyperoxia or by reducing leukocyte chemotaxis. That we did not observe the same effect in follistatin-treated animals was unexpected and this may be due to insufficient dosage of follistatin since only one dosage was tested in this study. Dose studies using follistatin and specific inhibitors for the members of the TGF $\beta$ superfamily will be needed to resolve confounding data from follistatin treatment groups. Despite the absence of an effect on total macrophage numbers in the lungs, it may be useful to determine if inhibition of activin A affects macrophage polarization.

In vitro, activin A negatively regulates IL-10 production by murine M2 macrophages and impairs their acquisition of an anti-inflammatory phenotype and cytokine profile via Smad $2 / 3$ phosphorylation (27). This supports the role of activin A in inflammatory diseases including BPD since macrophage polarization is a relevant event during wound healing and resolution of inflammation. Understanding the regulation of activin signaling in a disease such as BPD is important since M2 macrophages are implicated in early lung development, where they

Table 1. Treatment groups

\begin{tabular}{lll}
\hline & $\mathrm{FiO}_{2}$ & Treatment/dosage \\
\hline Normoxia alone & 0.21 & Saline \\
Hyperoxia alone & 0.85 & Saline \\
Normoxia + ActRIIB-Fc & 0.21 & $5 \mathrm{mg} / \mathrm{kg}$ \\
Hyperoxia + ActRIIB-Fc & 0.85 & $5 \mathrm{mg} / \mathrm{kg}$ \\
Normoxia + follistatin & 0.21 & $0.1 \mathrm{mg} / \mathrm{kg}$ \\
Hyperoxia + follistatin & 0.85 & $0.1 \mathrm{mg} / \mathrm{kg}$ \\
\hline FiO $_{2}$ fraction of inspired oxygen. & &
\end{tabular}

localized to branching points of the developing mouse lung (28). Keeping in mind the potential benefits and detrimental effects on the developing lungs, developing therapies that target activin A signaling can thus be precarious. Certainly in our hands ActRIIB-Fc and follistatin treatments showed some beneficial effects to lung development but thorough studies into macrophage polarity, concurrent alveolarisation, and secondary septation are needed to ascertain the feasibility of such a therapeutic strategy.

Surprisingly, treatment with ActRIIB-Fc did not change neutrophil numbers in the lungs, given that adenoviral overexpression of activin A in adult mouse lungs increased neutrophil influx into the lungs with an accompanying "cytokine-storm" of MCP-1, IL-1 $\beta$, IL-4, IL-5, IL-6, IL-10, IL-17, TNF $\alpha$, and IFN $\gamma$ (7). However, the relationship between activin $A$ and neutrophils is very poorly understood. Indeed, there have been no studies describing how activin A influence the neutrophil chemotaxis into the lungs, nor the regulation of activin A production by neutrophils during lung injury.

Septal crest density was improved in ActRIIB-Fc-treated animals, suggesting that lung development is benefitted by reduced inflammation. Metalloproteinases contribute to the pathological remodeling in neonatal rats exposed to $100 \%$ oxygen (29). In this study, $85 \%$ oxygen did not significantly change MMP-2 activity, but MMP-9 activity was increased. These findings were similar to a previous study performed using $90 \%$ oxygen to induce BPD in neonatal rats (12). Increased levels of MMP-9 have also been reported in premature infants with antenatal lung inflammation preceding chronic lung disease (30).

Furthermore, the ratio of MMP-9 to its inhibitor, TIMP1 , is elevated in premature infants who develop BPD (31). Furthermore, MMP-9- mice were resistant to hyperoxiainduced BPD (32). Together, these findings indicate the role of MMP-9 in the destruction of lung architecture during BPD. We observed in this study that ActRIIB-Fc treatment restored MMP-9 activity levels comparable to normoxia animals. This reduction in MMP-9 activity, along with increased cell proliferation, may account for the improvement in secondary septal crest density and overall improvement in lung structure. Despite the absence of pre-exisiting data on the influence of activin A on MMP-9 activity, the inhibition of activin A signaling using follistatin was recently shown to prevent pathological airway remodeling in asthma (33).

Increase in total AEC2 cells following exposure to hyperoxia was recently reported by Yee et al. (34). In this study, we observed that ActRIIB-Fc treatment reduced the percentage of proliferating cells while keeping the percentage of proliferating AEC2 cells unchanged. This cell proliferation was not, however, primarily associated with increased proliferation of AEC2 as there was no significant increase in Ki67+SPC+ cells following ActRIIB-Fc treatment. Other proliferating cells that may have contributed to differences in Ki67+ numbers include lung fibroblasts, endothelial cells, and endogenous lung progenitors, all of which are recruited temporally during periods of development and repair $(6,35,36)$. 
Another possibility is that ActRIIB-Fc treatment reversed the detrimental effects of activin A on angiogenesis. The VEGF signaling pathway drives postnatal angiogenesis and alveolarisation, and this is dysregulated in BPD-affected infants where there pulmonary capillary density is markedly reduced (37). Similarly, hyperoxia-induced rat BPD results in reduced VEGF expression and rescuing angiogenesis restores alveolarisation (38). It should be noted, however, that the role of activin A in vascular formation and alveolarisation has never been studied in the lung, during normal or pathological development.

In this study, we observed that inhibition of activin A signaling provides some protective effects against hyperoxia-associated growth/weight retardation, pathological lung remodeling, and leukocyte infiltration. These findings correlated strongly to the reduction of Smad 3 phosphorylation in a manner similar to that reported in curcumin inhibition of TGF $\beta$ activity (5). The common signaling intermediates used by TGF $\beta$ and activin during hyperoxic lung injury suggest that activin may contribute to lung development and BPD-related pathology.

\section{METHODS}

\section{Animal Experiments}

All experimental procedures were approved by Monash University Animal Ethics Committee and were conducted in accordance with the Australian Code of Practice for the Care and Use of Animals for Scientific Purposes. We used a newborn hyperoxia lung injury model, as previously described (39). Briefly, C57Bl/6 female mice aged 8-10 wk were time-mated and acclimatized to plexiglass chambers (12-h day/night cycle at $22{ }^{\circ} \mathrm{C}$ and $50-60 \%$ humidity) at about $17 \mathrm{~d}$ gestation (term 19-21). Within $24 \mathrm{~h}$ of birth, whole litters were randomized to either normoxia $\left(21 \% \mathrm{O}_{2}\right)$ or hyperoxia $\left(85 \% \mathrm{O}_{2}\right)$ for $14 \mathrm{~d}$. To prevent maternal oxygen toxicity, nursing dams were rotated every 2 d.

\section{Experimental Groups}

Litters were randomly assigned to one of six groups (four litters per group): normoxia alone, hyperoxia alone, normoxia + ActRIIB-Fc, hyperoxia + ActRIIB-Fc, normoxia + follistatin, hyperoxia + follistatin. All treatments were administered in $20 \mu \mathrm{l}$ saline via intraperitoneal injections twice weekly. Detailed breakdown of these groups are shown in Table 1. The detailed production of soluble ActRIIB-Fc has been previously described (40) and used at $5 \mathrm{mg} / \mathrm{kg}$. Recombinant follistatin (FS288) was obtained from R\&D systems (Minneapolis, $\mathrm{MN}$ ) and used at $0.1 \mathrm{mg} / \mathrm{ml}$.

\section{Postmortem and Tissue Collection}

Animals were humanely killed at postnatal day 14 with sodium pentobarbitone (Virbac, Regents Park, NSW, Australia). Pups were randomly assigned for histological and molecular analyses. For histological analyses, lungs were instilled with $4 \%$ paraformaldehyde at $20 \mathrm{cmH}_{2} \mathrm{O}$ pressure and immersed in $4 \%$ paraformaldehyde for $24 \mathrm{~h}$ before processing. For molecular analyses, lungs were either snapfrozen with liquid nitrogen or placed in RNAlater (Life Technologies, Carlsbad, CA) and stored at $-80^{\circ} \mathrm{C}$.

\section{Immunohistochemistry and Image Analysis}

Lungs were cut to a thickness of $5 \mu \mathrm{m}$. Sections were stained with hematoxylin and eosin or Hart's stain to determine tissue-airspace ratio and septal crest density, scoring 10 fields of view (five per individual lung) at $400 \times$ magnification. Briefly, the area fraction of positive tissue was quantified by digital image analysis using Image J software (NIH, Bethesda, MA). Each image was converted to a 16-bit black-and-white image and threshold values adjusted to include only stained areas. Threshold values were maintained for all samples. The active area score was defined as the percentage of stained pixels per field of view. Ten fields of view (five per individual lung) were assessed at $400 \times$ magnification.

To assess numbers of total leukocytes, neutrophils and macrophages, tissue sections were stained with antibodies against CD45 (560501, 1:200, BD Biosciences, Franklin Lakes, NJ), myeloperoxidase (ab45977, 1:200, Abcam, Cambridge, UK), or F4/80 (MCA 497R, $1: 200$, Serotec, Oxford, UK) respectively as previously described. The percentage of $\mathrm{CD}^{2} 5^{+}, \mathrm{MPO}^{+}, \mathrm{F} 4 / 80^{+}$cells was determined by Image J analysis (NIH). Ten fields of view (five per lung) were assessed at $400 \times$ magnification. Double staining of proliferating type II alveolar epithelial cells was performed using antibodies against Ki67 (ab15580, 1:250, Abcam) and surfactant protein C (SPC, sc-7705, 1:200, Santa Cruz Biotechnology, Santa Cruz, CA) and appropriate fluorescent secondary antibodies (Life Technologies).

\section{Protein Analyses}

Protein concentration of lung lysates was determined by BCA assay (Thermo Fisher, Waltham, MA). Forty micrograms of total protein were loaded into each well of a $4-12 \%$ bis-tris polyacrylamide gel under reducing conditions prior to transfer onto a polyvinylidene fluoride membrane. The membranes were blocked with $5 \%$ bovine serum albumin before probing with antibodies against total and phosphorylated Smad 3 (\#9513, 1:200 and C25A9, 1:200, respectively) and appropriate secondary biotinylated secondary antibodies. All antibodies were purchased from Cell Signaling Technology (Danvers, MA). Bands were visualized using Supersignal West Pico chemiluminescent substrate (Thermo Fisher) and quantified using a Chemidoc XRS+ (Biorad, Hercules, CA). Phosphorylation of Smad 3 was expressed as a ratio to total Smad 3. Activin A and IL-6 enzymelinked immunosorbent assay was performed according to manufacturer's instructions (SEA001Mu, Life Research, Scoresby, Victoria, Australia and R\&D Systems).

\section{Statistical Analysis}

Data were analyzed using GraphPad Prism 5 for Mac OS X (GraphPad Software, La Jolla, CA). Data are expressed as means \pm SEM. One-way ANOVA and post hoc Bonferroni's test were used for multiple group comparisons. Statistical significance was accorded for $P$ values $<0.05$.

\section{STATEMENT OF FINANCIAL SUPPORT}

The authors acknowledge financial support from the Victorian Government's Operational Infrastructure Support Program.

Disclosure: The authors have nothing to disclose.

\section{REFERENCES}

1. Northway WH Jr, Rosan RC, Porter DY. Pulmonary disease following respirator therapy of hyaline-membrane disease. Bronchopulmonary dysplasia. N Engl J Med 1967;276:357-68.

2. Jobe AH, Bancalari E. Bronchopulmonary dysplasia. NICHD/NHLBI/ ORD Workshop Summary. Am J Resp Crit Care Med 2001;163:1723-9.

3. Kallapur SG, Jobe AH. Contribution of inflammation to lung injury and development. Arch Dis Child Fetal Neonatal Ed 2006;91:F132-5.

4. Buckley S, Shi W, Barsky L, Warburton D. TGF-beta signaling promotes survival and repair in rat alveolar epithelial type 2 cells during recovery after hyperoxic injury. Am J Physiol Lung Cell Mol Physiol 2008;294: L739-48.

5. Sakurai R, Li Y, Torday JS, Rehan VK. Curcumin augments lung maturation, preventing neonatal lung injury by inhibiting TGF- $\beta$ signaling. Am J Physiol Lung Cell Mol Physiol 2011;301:L721-30.

6. Rehan VK, Sakurai R, Corral J, et al. Antenatally administered PPARgamma agonist rosiglitazone prevents hyperoxia-induced neonatal rat lung injury. Am J Physiol Lung Cell Mol Physiol 2010;299:L672-80.

7. Apostolou E, Stavropoulos A, Sountoulidis A, et al. Activin-A overexpression in the murine lung causes pathology that simulates acute respiratory distress syndrome. Am J Respir Crit Care Med 2012;185:382-91.

8. Aoki F, Kurabayashi M, Hasegawa Y, Kojima I. Attenuation of bleomycininduced pulmonary fibrosis by follistatin. Am J Respir Crit Care Med 2005;172:713-20. 
9. Dasgupta C, Sakurai R, Wang Y, et al. Hyperoxia-induced neonatal rat lung injury involves activation of TGF- $\{$ beta $\}$ and Wnt signaling and is protected by rosiglitazone. Am J Physiol Lung Cell Mol Physiol 2009;296:L1031-41.

10. Kunzmann S, Speer CP, Jobe AH, Kramer BW. Antenatal inflammation induced TGF-betal but suppressed CTGF in preterm lungs. Am J Physiol Lung Cell Mol Physiol 2007;292:L223-31.

11. Madurga A, Mižíková I, Ruiz-Camp J, et al. Systemic hydrogen sulfide administration partially restores normal alveolarization in an experimental animal model of bronchopulmonary dysplasia. Am J Physiol Lung Cell Mol Physiol 2014;306:L684-97.

12. Buckley S, Warburton D. Dynamics of metalloproteinase-2 and -9, TGFbeta, and uPA activities during normoxic vs. hyperoxic alveolarization. Am J Physiol Lung Cell Mol Physiol 2002;283:L747-54.

13. Yee M, Buczynski BW, Lawrence BP, O’Reilly MA. Neonatal hyperoxia increases sensitivity of adult mice to bleomycin-induced lung fibrosis. Am J Respir Cell Mol Biol 2013;48:258-66.

14. Chen H, Zhuang F, Liu YH, et al. TGF-beta receptor II in epithelia versus mesenchyme plays distinct roles in the developing lung. Eur Respir J 2008;32:285-95.

15. Chen H, Sun J, Buckley S, et al. Abnormal mouse lung alveolarization caused by Smad3 deficiency is a developmental antecedent of centrilobular emphysema. Am J Physiol Lung Cell Mol Physiol 2005;288:L683-91.

16. Alejandre-Alcázar MA, Kwapiszewska G, Reiss I, et al. Hyperoxia modulates TGF-beta/BMP signaling in a mouse model of bronchopulmonary dysplasia. Am J Physiol Lung Cell Mol Physiol 2007;292:L537-49.

17. Glister C, Kemp CF, Knight PG. Bone morphogenetic protein (BMP) ligands and receptors in bovine ovarian follicle cells: actions of BMP-4, -6 and -7 on granulosa cells and differential modulation of Smad-1 phosphorylation by follistatin. Reproduction 2004;127:239-54.

18. Sako D, Grinberg AV, Liu J, et al. Characterization of the ligand binding functionality of the extracellular domain of activin receptor type IIb. J Biol Chem 2010;285:21037-48.

19. Nüsing RM, Barsig J. Induction of prostanoid, nitric oxide, and cytokine formation in rat bone marrow derived macrophages by activin $\mathrm{A}$. Br J Pharmacol 1999;127:919-26.

20. Brosh N, Sternberg D, Honigwachs-Sha'anani J, et al. The plasmacytoma growth inhibitor restrictin-P is an antagonist of interleukin 6 and interleukin 11. Identification as a stroma-derived activin A. J Biol Chem 1995;270:29594-600.

21. Wang SY, Tai GX, Zhang PY, Mu DP, Zhang XJ, Liu ZH. Inhibitory effect of activin A on activation of lipopolysaccharide-stimulated mouse macrophage RAW264.7 cells. Cytokine 2008;42:85-91.

22. Segerer SE, Müller N, Brandt Jv, et al. The glycoprotein-hormones activin A and inhibin A interfere with dendritic cell maturation. Reprod Biol Endocrinol 2008;6:17.

23. Huber S, Stahl FR, Schrader J, et al. Activin a promotes the TGFbeta-induced conversion of CD4+CD25- T cells into Foxp3+ induced regulatory T cells. J Immunol 2009;182:4633-40.

24. Semitekolou M, Alissafi T, Aggelakopoulou M, et al. Activin-A induces regulatory $\mathrm{T}$ cells that suppress $\mathrm{T}$ helper cell immune responses and protect from allergic airway disease. J Exp Med 2009;206:1769-85.
25. Ogawa K, Funaba M, Chen Y, Tsujimoto M. Activin A functions as a Th2 cytokine in the promotion of the alternative activation of macrophages. J Immunol 2006;177:6787-94.

26. Ohga E, Matsuse T, Teramoto S, Ouchi Y. Activin receptors are expressed on human lung fibroblast and activin A facilitates fibroblast-mediated collagen gel contraction. Life Sci 2000;66:1603-13.

27. Sierra-Filardi E, Puig-Kröger A, Blanco FJ, et al. Activin A skews macrophage polarization by promoting a proinflammatory phenotype and inhibiting the acquisition of anti-inflammatory macrophage markers. Blood 2011;117:5092-101.

28. Jones CV, Williams TM, Walker KA, et al. M2 macrophage polarisation is associated with alveolar formation during postnatal lung development. Respir Res 2013;14:41.

29. Pardo A, Selman M, Ridge K, Barrios R, Sznajder JI. Increased expression of gelatinases and collagenase in rat lungs exposed to $100 \%$ oxygen. Am J Respir Crit Care Med 1996;154(4 Pt 1):1067-75.

30. Curley AE, Sweet DG, Thornton CM, et al. Chorioamnionitis and increased neonatal lung lavage fluid matrix metalloproteinase-9 levels: implications for antenatal origins of chronic lung disease. Am J Obstet Gynecol 2003;188:871-5.

31. Sweet DG, Curley AE, Chesshyre E, et al. The role of matrix metalloproteinases -9 and -2 in development of neonatal chronic lung disease. Acta Paediatr 2004;93:791-6.

32. Chetty A, Cao GJ, Severgnini M, Simon A, Warburton R, Nielsen HC. Role of matrix metalloprotease-9 in hyperoxic injury in developing lung. Am J Physiol Lung Cell Mol Physiol 2008;295:L584-92.

33. Hardy CL, Nguyen HA, Mohamud R, et al. The activin A antagonist follistatin inhibits asthmatic airway remodelling. Thorax 2013;68:9-18.

34. Yee M, Buczynski BW, Lawrence BP, O’Reilly MA. Neonatal hyperoxia increases sensitivity of adult mice to bleomycin-induced lung fibrosis. Am J Respir Cell Mol Biol 2013;48:258-66.

35. Rock JR, Hogan BL. Epithelial progenitor cells in lung development, maintenance, repair, and disease. Annu Rev Cell Dev Biol 2011;27:493-512.

36. Thébaud B. Angiogenesis in lung development, injury and repair: implications for chronic lung disease of prematurity. Neonatology 2007;91:291-7.

37. Bhatt AJ, Pryhuber GS, Huyck H, Watkins RH, Metlay LA, Maniscalco WM. Disrupted pulmonary vasculature and decreased vascular endothelial growth factor, Flt-1, and TIE-2 in human infants dying with bronchopulmonary dysplasia. Am J Respir Crit Care Med 2001;164(10 Pt 1):1971-80.

38. Thébaud B, Ladha F, Michelakis ED, et al. Vascular endothelial growth factor gene therapy increases survival, promotes lung angiogenesis, and prevents alveolar damage in hyperoxia-induced lung injury: evidence that angiogenesis participates in alveolarization. Circulation 2005;112: 2477-86.

39. Vosdoganes P, Lim R, Koulaeva E, et al. Human amnion epithelial cells modulate hyperoxia-induced neonatal lung injury in mice. Cytotherapy 2013;15:1021-9.

40. Hoogaars WM, Mouisel E, Pasternack A, et al. Combined effect of AAVU7-induced dystrophin exon skipping and soluble activin Type IIB receptor in mdx mice. Hum Gene Ther 2012;23:1269-79. 